\title{
Motivations and values associated with combining sex and illicit drugs ('chemsex') among gay men in South London: findings from a qualitative study
}

Weatherburn $\mathrm{P}(\mathrm{MSc}, \mathrm{BA})^{1}$, Hickson $\mathrm{F}(\mathrm{PhD}, \mathrm{BA}, \mathrm{BSc})^{1}$, Reid D (MSc, BSc) ${ }^{1}$, Torres-Rueda $\mathrm{S}$ $(\mathrm{MSc}, \mathrm{BA})^{2}$, Bourne $\mathrm{A}(\mathrm{PhD}, \mathrm{BSc})^{1 *}$

${ }^{1}$ Sigma Research, Department of Social \& Environmental Health Research, London School of Hygiene \& Tropical Medicine.

${ }^{2}$ Department of Global Health and Development, London School of Hygiene \& Tropical Medicine.

* Corresponding author:

Adam Bourne

Sigma Research

London School of Hygiene \& Tropical Medicine

15-17 Tavistock Place, London, WC1H 9SH

Email: adam.bourne@Ishtm.ac.uk

Tel: 020-7927-2793

\footnotetext{
"The Corresponding Author has the right to grant on behalf of all authors and does grant on behalf of all authors, an exclusive licence (or non exclusive for government employees) on a worldwide basis to the BMJ Publishing Group Ltd to permit this article (if accepted) to be published in STI and any other BMJPGL products and sub-licences such use and exploit all subsidiary rights, as set out in our licence

http://group.bmj.com/products/journals/instructions-for-authors/licence-forms".
}

MeSH terms: sexual behavior; illicit drugs; HIV; qualitative research; London

Word count (Introduction to end of Discussion): $\underline{3016}$ 


\section{Abstract}

Objectives: There is considerable public health concern about the combining of sex and illicit drugs (chemsex) among gay men. With a view to informing supportive therapeutic and clinical interventions, we sought to examine the motivations for engaging in chemsex among gay men living in South London.

Methods: Community advertising recruited 30 gay men for qualitative semi-structured interview. Aged between 21 and 53 years, all lived in South London in the boroughs of Lambeth, Southwark and Lewisham and all had combined crystal methamphetamine, mephedrone and/or GHB/GBL with sex in the past 12 months. Transcripts were subjected to a thematic analysis.

Results: We broadly distinguish two groups of reasons for combining sex and drugs, within which we describe eight distinct motivations. The first major group of motivations for combining drugs with sex is that drugs provide the means by which men can have the sex they desire by increasing libido, confidence, disinhibition and stamina. The second major group of motivations for chemsex is that drugs enhance the qualities of the sex that men value. Drugs made other men seem more attractive, increased physical sensations, intensified perceptions of intimacy and facilitated a sense of sexual adventure.

Conclusion: Analysis revealed that sexualised drug use provides both motivation and capability to engage in the kinds of sex that some gay men value: sex that explores and celebrates adventurism. Those services providing (talking) interventions to men engaging in chemsex should consider these benefits of sexualised drug use alongside the harms arising.

MeSH terms: sexual behaviour; illicit drugs; risk; HIV; qualitative research; London 


\section{Introduction}

Throughout the 1990s and early 2000s the illicit drugs most commonly used by MSM were cannabis (a depressant) and three stimulants: amphetamine (speed), methylene-dioxymeth-amphetamine (MDMA, or ecstasy) and cocaine. $(1,2)$ Over the last decade, academic research $(3,4)$, clinic data $(5)$ and the gay media $(6,7)$ have charted the rise of two other stimulants: methyl-meth-cathinone (mephedrone) and crystal meth-amphetamine (crystal meth). In addition, another depressant has also emerged: gamma-hydroxy-butyric acid ('GHB', sometimes taken as the pro-drug gamma-butyrol-actone or ' $G B L^{\prime}$ ). These three newer drugs have given rise to a phenomenon commonly referred to as "chemsex" (8-10). Recent evidence (11) suggests $6.6 \%$ of gay men and bisexual men in England had used any of these three core chemsex drugs in the last 4 weeks. The figure was $14.3 \%$ for men living in London, 21.9\% for men living with diagnosed HIV in England, and 32.7\% for men living with diagnosed HIV in London.

Chemsex is a linguistic and sociological category loosely defined around the intentional combining of sexual activity and consumption of illicit psychoactive substances, in particular crystal meth, mephedrone and $\operatorname{GHB} / \operatorname{GBL}(10,12)$. While polydrug use - the use of two or more drugs within the same session - chemsex sessions do not necessarily include all these three drugs, and many men engaged in chemsex also use other drugs (especially ketamine, ecstasy and cocaine) (13). Chemsex is not a unitary behaviour but a diverse set of sexual and drug-taking behaviours in complex interactions. Chemsex can include just two participants but often includes more, and can take place in private homes (often facilitated by geospatial sexual networking apps) or in gay specific sex-on-premises venues $(9,13)$.

Illicit drugs (especially crystal methamphetamine) have been shown to be associated with condomless intercourse at both the individual (14) and sexual session (15) levels. Although not all studies find this association, (16) and although causality is difficult to demonstrate (17) it is clear that chemsex is rarely safer than sober sex. (8)

Understanding the sources of behaviour is a pre-requisite of successful health promotion interventions. Michie and colleagues(18) have developed a consensus-based typology of the varying accounts of behaviours and activities to change them, in order to better plan and 
evaluate interventions. They demarcate three categories of sources of behaviour: opportunity, capability and motivation.

Opportunities include the physical and social frameworks that make a behaviour possible. In the case of chemsex these might include the availability of drugs, the physical spaces in which to engage (eg. homes, sex-on-premises-venues), the new methods of contact that enable social connections like the geo-spatial mobile phone app. (8)

Sexual behaviour is learnt, both in terms of social norms and procedures and also physical acts and techniques. Capabilities refer to the psychological and physical capacities required to carry out a behaviour, which range from self-confidence to participate in group sex to the ability to maintain an erection over a very long sexual session. Focus groups of gay men using crystal meth at gay circuit parties in Miami highlighted the instrumental use of drugs in reducing body image anxiety, losing sexual inhibitions and facilitating social and sexual connection.(19) Drugs can be used to remove the obstacles that make men less able to have the sex they want.

Finally, motivation refers to the internal driver for a behaviour. Motivations may be automatic and unmediated by conscious thought, or reflective and involve taking a decision and forming an intention. In the absence of any published data relating to motivations to use drugs during sex among gay men, this paper describes findings from a series of qualitative in-depth interviews with gay men living in South London. By closely examining the factors that men value about sexualised drug use, we begin to build a picture of their motivations for chemsex. Understanding the motivations for chemsex, especially the benefits men perceive, is crucial to inform psychotherapeutic and clinical services that aim to support gay men to minimise harm while maximising sexual pleasure.

\section{Methods}

With a view to informing therapeutic and clinical interventions, we aimed to examine the motivations for engaging in chemsex among gay men living in South London using qualitative research methods. 
Between August 2013 and February 2014 we recruited 30 men living in the London boroughs of Lambeth, Southwark or Lewisham (LSL) who engaged in chemsex with other men, for in-depth qualitative interviews. These three boroughs have large gay populations and a high prevalence of diagnosed HIV. (20) The eligibility criteria were: being male and over 18 years of age; living in LSL; and having used crystal methamphetamine, mephedrone or $\mathrm{GHB} / \mathrm{GBL}$ during sex with another man at least once during the previous 12 months. We placed paid advertisement in a gay-scene magazine and on geo-spatial sexual networking applications, sought community-based organisation referrals and distributed promotional cards in gay venues. While it was a convenience sample of men engaging in chemsex we sought, as far as possible, to balance the sample of participants according to HIV status, ethnicity and borough of residence.

All promotional materials directed potential participants to a webpage containing a project description and an invitation to contact the lead researcher. All participants self-identified as gay, with a mean age was 36 years (range 21-53). Thirteen were living with diagnosed HIV. Sixteen identified as White British, 11 as members of other white groups and three were from ethnic minorities.

Private semi-structured, one-to-one, face-to-face interviews (1-2 hours) were conducted by $A B$ and $D R$ (who have combined qualitative interview experience of more than 30 years) at the study centre or in participants' homes. Following discussion of the study aims and informed consent, interviews focussed on men's history of drug use, the impact of drugs on their sexual behaviour, their experiences and perceptions of harm, and of accessing support in relation to their drug use. Interviews were digitally recorded, transcribed verbatim and thematically analysed.(21)

Data were read, and initial codes were documented, organised into potential themes and all examples of each theme were recorded. For this specific analysis, identification of key themes was undertaken by one author (PW) and corroborated by two others (DR and $A B$ ). Typical quotes were identified to illustrate themes (shown here in italics, with participant's age). 


\section{Findings}

We broadly distinguish two forms of motivation for combining sex and illicit drugs within which we describe eight distinct motivations. These benefits of sexualised drug use arise from interviews with men that had combined crystal methamphetamine, mephedrone and/or GHB/GBL with sex in the past 12 months. While we focussed on these three chemsex drugs substantially, some men also described sex under the influence of other drugs, most commonly ketamine, ecstasy or cocaine.

\section{Providing the capability for the sex that is wanted}

The first major motivation for combining drugs with sex is that drugs provide the means by which men can have the sex they desire. Men used drugs instrumentally to meet several of the requirements for having the kind of sex they wanted, including libido, confidence, disinhibition, and stamina.

Increasing arousal and restoring libido

All of the men we interviewed spoke of drugs as increasing their libido or making them feel sexually aroused. Men often struggled to articulate this sensation in any greater detail, but some described their increased sex drive under the influence of drugs in very emotive terms, suggesting that it was insatiable or overpowering. Crystal meth, especially when injected, was notable for this effect.

"I don't have a sex drive any longer. It's one of the reasons why I started slamming chems because when I slam, I get horny." [Aged 53, diagnosed HIV positive]

In a somewhat paradoxical manner, libido was usually understood to be the motivation for sexual behaviour. Men found themselves wanting to be 'in the mood' for sex but not feeling so. They were (reflectively) motivated to use drugs because they provide the (automatic) motivation to engage in sex by creating or awakening sexual arousal. On the other hand, many men later described a range of sexually inhibiting experiences (pain, tiredness, doubt, hesitancy; see below), which when alleviated by drugs would allow their sexual desire to activate. 
Increasing sexual confidence

One highly valued effect of these drugs was their ability to enhance self-confidence and inhibit self-consciousness and doubt. Many participants described current or past sexual insecurities which had a detrimental impact on their ability to have enjoyable sex. These feelings had diverse and complex origins including concerns relating to internalised homophobia, coping with an HIV diagnosis, guilt related to having or wanting sex with men, and negative body-image and/or feeling unattractive and unworthy as an object of desire for other men.

I think when I was using drugs I did not have body issues. I did not think, I am feeling a bit too fat or feeling that I do not really feel that attractive so it reduces inhibitions physically and psychologically in terms of having sex and with people you would not feel comfortable, like, having sex with normally. [Aged 40, diagnosed HIV positive]

Men also described how drugs enhance sexual confidence by moderating the fear of rejection and ameliorating its effects. Men who normally lacked self-confidence or selfesteem frequently worried about whether others would be sexually or romantically interested in them. This acted as a barrier to engaging in conversation or sexual contact because the perceived probability of rejection was high. Drugs served both to remove this cognitive barrier and lessen the pain if rejection did occur.

Several participants also expressed concern that they were not as "good at sex" as they wanted to be and that knowing that drugs would provide sexual confidence and enhance performance, they were less anxious about having sex if drugs were involved.

\section{Losing inhibitions}

Almost every man described drugs as helping them 'lose their inhibitions', often around specific sexual acts. Many were unable to articulate more than this common substance use discourse. However, when prompted, this man said:

What I mean by, 'losing my inhibitions', is you certainly don't hold back. You don't - I don't have that moment of thinking, 'Oh, should I? Shouldn't I?' [...] It's like - you want something; you get it. [Aged 28, diagnosed HIV positive] 
Many men wanted to engage in a wider range of sexual activity than their inhibitions would allow when sober; drugs allowed them to do so (see Facilitating sexual adventure and stimulation below). While under the influence of drugs, competing cognitions (both aversive responses and personally held limits) were often disregarded.

Increasing sexual longevity

Some participants valued long sexual sessions. Drugs facilitated sexual stamina, and men reported being able to have sex for long periods of time without ejaculating, and/or being ready to have sex again very quickly after ejaculating. This effect was particularly pronounced among those using crystal meth, who reported that sexual sessions could last for many hours or, in some cases, days.

"So for me and this guy, we would smoke it [crystal] together and literally just fuck him for 12 hours, non-stop and it was brilliant. It was just-you feel super human, he can take it and take it. You give it and give it." [Aged 40, last tested HIV negative]

Around a third of participants described recent chemsex experiences involving sex with numerous men, over an extended period of time (often 24 hours or more), sometimes in several different locations. Taking drugs throughout this period meant they could maintain high levels of both energy and desire, even if they sometimes experienced problems with erections. Drugs facilitated high turnover of sexual partners by enabling longer sexual sessions and because they were used in highly sexualised environments (such as saunas and sex parties) where multi-partner sex is common.

\section{Enhancing the qualities valued in sex}

The second major motivation for chemsex was that drugs were seen to enhance the qualities of the sex that men valued. Drugs made other men seem more attractive, heightened physical sensations, intensified perceptions of intimacy and facilitated sexual adventure.

\section{Enhancing sexual attraction}

Some men described how their perceptions of the attractiveness of potential partners were greatly enhanced when they were using drugs. 
Within ten to fifteen minutes of taking it [mephedrone] the world is a prettier place, so everybody becomes more attractive. People have got bigger muscles, bigger penises, their legs are more powerful. [Aged 50, last tested HIV negative]

The enhancement of sexual incentives is a common effect of a range of drugs, including alcohol (often colloquially termed "beer goggles"). This is not a hallucination but an enhancement or sensitisation of the sexual response system.

\section{Intensifying sexual sensations}

For almost all participants, drugs could significantly enhance the physical sensation of sex. Men described intense physical stimulation, heightened arousal and orgasms that they never experienced sober. This was highly valued and explained why many used drugs for all, or nearly all, of their sexual experiences. This participant typifies the superlatives used:

[On mephedrone] It was the best sex I ever had. Really the best orgasm I'd had. I used to say it was like the heavens opened and it was like the light came down when I had an orgasm. Because it was that intense on drugs, it really was. I've never experienced that sober. [Aged 21, last tested HIV negative]

Experiencing this sensation forms a strong positive association between drugs and sex that then provides the automatic and reflective motivation to combine sex and drugs in the future.

\section{Enhancing emotional intimacy and sexual connection}

Valuing intimacy in sexual encounters was common among interviewees and over twothirds described how drugs were able to enhance the sense of connection they felt with their sexual partner. Casual sex often carried an emotional component and drugs provided more instant access to feelings of emotional closeness. Several participants articulated intense feelings of sexual intimacy and a sense that they were 'on another level' with their sexual partner. This stemmed from a feeling they were in touch with both their own senses and the desires of their partner.

However, achieving 'the same level' was a challenge. If the other person had taken different drugs, or more (or less) of a drug, then some reported it was difficult to achieve this 
connection. Also, an enhanced perception of intimacy or sexual connection was not a key factor in chemsex motivation for all participants - some felt that emotionally intimate sex was not possible on drugs.

\section{Facilitating sexual adventure and stimulation}

Chemsex was universally described as more intense and adventurous than sex without drugs. On drugs, men commonly engaged in a greater diversity of sexual acts, such as group sex, use of toys, bondage and domination, graphic talk, role playing and watersports (urolagnia). Many men also reported a greater likelihood of engaging in fisting (ano-brachial intercourse) while taking drugs, particularly crystal meth (but also ketamine). Those who injected, especially crystal meth, often felt that this delivery mechanism facilitated more "extreme" sexual acts.

Crystal meth, when you inject it, it just feels very dirty. Just very sleazy. All your inhibitions just lower [...] You do stuff that you wouldn't normally do, you would be into different fetishes that you probably wouldn't usually be in to." [Aged 24, diagnosed HIV positive]

Pre-existing limits of sexual expression were person-specific but there was general agreement that drugs encouraged the pushing of boundaries to try new, and potentially more extreme, activities. Participants felt it was much more likely they would try to enact sexual fantasies when using drugs. This was the case with both casual and regular partners with several of those in longer-term relationships describing how drug use added excitement to a familiar, and perhaps staid, sexual setting.

\section{Discussion}

Our analysis highlights eight overlapping themes that begin to describe the key pleasures and benefits of chemsex. These can be summarised into two meta-themes - increasing the capability to have the sex they wanted (increasing arousal and libido, increasing sexual confidence, reducing inhibitions, and increasing sexual stamina) and enhancing the qualities valued in sex (increasing sexual attraction, intensifying sensations, increasing intimacy and facilitating sexual adventure). 
Drugs provide both motivation (by increasing libido) and capability (by reducing inhibitions and by increasing confidence and stamina) for gay men to engage in the kinds of sex they value. In this regard, our findings echo those of previous qualitative investigations of drug use among gay men in the USA, France and Australia, which have highlighted the facilitative and enhancement role of drugs in sex (Kurtz, 2005) and the central place of the desires for pleasure (22) and excess.(23)

Alongside the transgressive account of chemsex (24), it is important to recognise that for some gay men, chemsex allows a different kind of liberation - from their own insecurities and anxieties about their body shape and sexual appeal; about being intimate with men; and having the kinds of sex they want, in the volume they desire. Chemsex allows many to transcend their own insecurities but it does not resolve them. Sober sex does not become more appealing after engaging in chemsex, and certainly becomes no easier to achieve. As a consequence, some gay men report a normalising of chemsex, and increased difficulty in forming and maintaining emotionally engaged, sober relationships.

The benefits of and motivations for chemsex we have identified have associated costs and potential harms, which we have described in detail elsewhere. $(8,12)$ While most men are not naïve to these harms, they sometimes lacked understanding of how to manage them, especially when their involvement in chemsex challenged their boundaries about physical and /or sexual safety.

While tailored chemsex support interventions have begun to emerge in several specialist London clinics, many gay men have struggled to access interventions that offered appropriate harm reduction and recovery support that was culturally appropriate and acceptable. (12) It is crucial that supportive services are further developed and that these take account of the diverse motivations for engagement in chemsex and recognise what some gay men value in the context of sexualised drug use. While it is possible that chemsex may facilitate an environment in which sexually transmitted infections (STIs) may be transmitted more readily, (8) culturally sensitive health promotion interventions need to recognise the reasons why individuals might wish to use drugs to have sex, in order to be acceptable and effective. 


\section{Key messages}

- For some gay men, drugs provide both motivation and capability to engage in the kinds of sex they value: sex that explores and celebrates adventurism.

- Chemsex can increase mens' capability to have the sex they want by increasing sexual arousal and libido, and sexual confidence and stamina and by reducing inhibitions.

- Chemsex can also enhance the qualities valued in sex by increasing sexual attraction and perceptions of intimacy, intensifying sensations and by facilitating sexual adventure.

- To be acceptable and effective services must take account of motivations for chemsex when seeking to help men avoid and mitigate the harms arising.

\section{Acknowledgements}

We wish to acknowledge the support of all those organisations and venues that helped to recruit participants for the study and those who kindly hosted interviews. Thanks to Paul Steinberg of Lambeth Council for ensuring the commission of this study and his thoughtful contribution throughout the process, and to the $\mathbf{3 0}$ men who gave up their time to share their experiences.

\section{Contributors}

$A B$ designed and led the study. $D R$ and $A B$ conducted the interviews. $A B, D R, S T R$ and PW contributed to primary data analysis. PW prepared the first draft of the manuscript. FH and $A B$ revised the manuscript. All authors commented on and approved the final version of this paper.

\section{Funding}

This research was commissioned and funded by the London boroughs of Lambeth, Southwark and Lewisham.

\section{Competing interests}

None declared. 


\section{Ethics approval}

Research Ethics Committee of the London School of Hygiene \& Tropical Medicine.

\section{References}

1. Keogh P, Reid D, Bourne A, Weatherburn P, Hickson F, Jessup K, et al. Wasted opportunities: problematic alcohol and drug use among gay men and bisexual men. London: Sigma Research, 2009.

2. Weatherburn P, Stephens M, Reid D, Hickson F, Henderson L, Brown D. Vital Statistics: Findings from the National Gay Men's Sex Survey, 1999. London: Sigma Research, 2000.

3. Bonell CP, Hickson FC, Weatherburn P, Reid DS. Methamphetamine use among gay men across the UK. Int J Drug Policy. 2010;21(3):244-6.

4. Measham F, Wood D, Dargan P, Moore K. The Rise in Legal Highs: Prevalence and patterns in the use of illegal drugs and first and second generation 'legal highs' in south London gay dance clubs. . Journal of Substance Use. 2011;16(4):263-72.

5. Stuart D. Sexualised drug use by MSM: background, current status and response. HIV Nursing. 2013;13(1):6-10.

6. Hopkins A. Chemsex revealed: Part 1. Beige Magazine. 2014 18th February 2014.

7. Marsh J. Sex, drugs and gay identity. QX Magazine. 2012.

8. Bourne A, Reid D, Hickson F, Torres-Rueda S, Weatherburn P. Illicit drug use in sexual settings ('chemsex') and HIV/STI transmission risk behaviour among gay men in South London: findings from a qualitative study. Sex Transm Infect. 2015;91(8):564-8.

9. Gilbart VL, Simms I, Jenkins C, Furegato M, Gobin M, Oliver I, et al. Sex, drugs and smart phone applications: findings from semistructured interviews with men who have sex with men diagnosed with Shigella flexneri $3 a$ in England and Wales. Sex Transm Infect. 2015;91(8):598-602.

10. McCall H, Adams N, Mason D, Willis J. What is chemsex and why does it matter? BMJ. 2015;351:h5790.

11. Hickson F, Reid D, Hammond G, Weatherburn P. State of play: findings from the England gay men's sex survey 2014. London: Sigma Research, London School of Hygiene \& Tropical Medicine, 2016.

12. Bourne A, Reid D, Hickson F, Torres-Rueda S, Steinberg P, Weatherburn P. "Chemsex" and harm reduction need among gay men in South London. Int J Drug Policy. 2015;26(12):1171-6.

13. Bourne A, Reid D, Hickson F, TorresRueda S, Weatherburn P. The Chemsex study: drug use in sexual settings among gay and bisexual men in Lambeth, Southwark and Lewisham. London: Sigma Research, London School of Hygiene \& Tropical Medicine, 2014.

14. Bolding G, Hart G, Sherr L, Elford J. Use of crystal methamphetamine among gay men in London. Addiction. 2006;101(11):1622-30.

15. Melendez-Torres GJ, Hickson F, Reid D, Weatherburn P, Bonell C. Findings from withinsubjects comparisons of drug use and sexual risk behaviour in men who have sex with men in England. Int J STD AIDS. 2016.

16. Prestage G, Fogarty AS, Rawstorne P, Grierson J, Zablotska I, Grulich A, et al. Use of illicit drugs among gay men living with HIV in Sydney. AIDS. 2007;21 Suppl 1:S49-55. 
17. Melendez-Torres GJ, Bourne A. Illicit drug use and its association with sexual risk behaviour among MSM: more questions than answers? Curr Opin Infect Dis. 2016;29(1):58-63.

18. Michie S, van Stralen MM, West R. The behaviour change wheel: a new method for characterising and designing behaviour change interventions. Implement Sci. 2011;6:42.

19. Kurtz SP. Post-circuit blues: motivations and consequences of crystal meth use among gay men in Miami. AIDS Behav. 2005;9(1):63-72.

20. Yin Z, Brown AE, Hughes G, Nardone A, Gill ON, Delpech VC. HIV in the United Kingdom 2014 Report: data to end 2013. London: Public Health England, 2014.

21. Braun V, Clarke V. Using thematic analysis in psychology. Qualitative Research in Psychology. 2006;3:77-101.

22. Gaissad L. Expending ourselves at "La Démence"? Gay party circuit from consumption to consummation. Ethnologie française 2013;43:409-16.

23. Hurley M, Prestage G. Intensive sex partying amongst gay men in Sydney. Cult Health Sex. 2009;11(6):597-610.

24. Halliday J, Godfrey C. NHS urged to respond to growing health dangers. The Guardian. 7th November 2015. 